\title{
Planificación microcurrícular de asignaturas en la profesión de enfermería y su vinculación con los modelos conceptuales
}

\author{
Microcurricular planning of subjects in the nursing profession and its link with conceptual \\ models
}

\section{Planejamento microcurricular de disciplinas da profissão de enfermagem e sua vinculação com modelos conceituais}

\author{
María Vera Márquez \\ mveram@utb.edu.ec \\ ORCID: 0000-0003-1486-9279
}

\author{
Esther Urdaneta de Primera \\ eurdaneta02@gmail.com \\ ORCID: 0000-0003-3245-2530
}

Universidad Técnica de Babahoyo, Ecuador

\begin{abstract}
RESUMEN
Introducción: El estudio tuvo como objetivo determinar desde la academia la inclusión y utilización de los modelos conceptuales, ligadas al proceso de atención, en las asignaturas relacionadas al cuidado dentro del microcurrículo, para verificar su nivel de inserción en la planificación curricular y transferencia de conocimientos al estudiante. Método: Se tipificó como descriptiva, de campo, transversal. La muestra estuvo constituida por 29 docentes del área profesionalizantes. Se diseñaron 2 instrumentos de recolección de datos: Ficha de registro de 12 sílabos para el análisis de los contenidos del cuidado con vinculación de teorizantes en y test a los docentes para confrontar su utilidad en las clases. Resultados: La revisión de los sílabos evidenció el 21,43\% incluyen teorizantes y el $78,57 \%$ no involucran en la planificación de las asignaturas, organización basada en patologías clínicas y no a diagnósticos enfermeros. La aplicación del test evidencia un dominio medio $63,06 \%$ en el conocimiento de los modelos conceptuales, un dominio medio $46,55 \%$ en el conocimiento y utilización de las teorizantes en el proceso de atención, 39,65\% corresponde al involucramiento de las teorizantes en los contenidos de las asignaturas seguido de un $31,03 \%$ con utilización bajo. Conclusiones: Se evidencia la poca inclusión de las teorizantes de enfermería, en el Proceso de Atención de Enfermería en la mayoría de las asignaturas de la unidad profesional y en las guías prácticas, lo que dificulta la relación teoría-practica del hacer enfermero.
\end{abstract}

Palabras clave: Educación en enfermería; docentes de enfermería; proceso de enfermería; modelos de enfermería

\begin{abstract}
Introduction: The study aimed to determine from the academy the inclusion and use of conceptual models, linked to the care process, in subjects related to care within the microcurriculum, to verify their level of insertion in curricular planning and knowledge transfer to the student. Method: It was classified as descriptive, field, cross-sectional. The sample consisted of 29 professors from the professional area. Two data collection instruments were designed: Record sheet of 12 syllables for the analysis of the contents of care with linking of theorists in and test the teachers to confront their usefulness in the classes. Results: The review of the syllables showed that $21.43 \%$ include theorizers and $78.57 \%$ do not involve organization based on clinical pathologies and not on nursing diagnoses in the planning of the subjects. The application of the test shows a $63.06 \%$ average domain in the knowledge of the conceptual models, a $46.55 \%$ average domain in the knowledge and use of theorizers in the care process, $39.65 \%$ corresponds to the involvement of the theorizing in the contents of the subjects followed by $31.03 \%$ with low use. Conclusions: The little inclusion of nursing theorists is evident, in the Nursing Care Process in most of the subjects of the professional unit and in the practical guides, which hinders the theory-practice relationship of becoming a nurse
\end{abstract}

Key words: Nursing education; nursing teachers; nursing process; nursing models

MV: Licenciada en Enfermería con Maestría en Gerencia de Servicios de Salud y Maestría en Docencia y Currículo. Actualmente Docente titular agregada. Directora de la Escuela de Enfermería y de la Escuela de Enfermería y de la Escuela Salud y Bienestar en la Facultad de Ciencias de la Salud. Universidad Técnica de Babahoyo, Ecuador.

EU: Licenciada en enfermería. Maestría en Administración de Hospitales y Doctorado en Ciencias Gerenciales. Trabajo en Docencia y área asistencial. Actualmente supervisora de Enfermería y profesor agregado en el Departamento de Gestión del Cuidado. Experiencia en área de investigación. Tutora y jurado de tesis pre y postgrado. Universidad Del Zulia. Venezuela. 
MV: Licenciada en Enfermería con Maestría en Gerencia de Servicios de Salud y Maestría en Docencia y Currículo. Actualmente Docente titular agregada. Directora de la Escuela de Enfermería y de la Escuela de Enfermería y de la Escuela Salud y Bienestar en la Facultad de Ciencias de la Salud. Universidad Técnica de Babahoyo, Ecuador.

EU: Licenciada en enfermería. Maestría en Administración de Hospitales y Doctorado en Ciencias Gerenciales Trabajo Docencia y área asistencial. Actual Docencia y área asistencial. Actualmente supervisora de Enfermería y profesor agregado en el Departamento de Gestión del Cuidado. Experiencia en área de investigación. Tutora y jurado de tesis pre y postgrado Universidad Del Zulia Universidad Del Zulia. Venezuela.

\section{RESUMO}

Introdução: O estudo objetivou determinar a partir da academia a inclusão e utilização de modelos conceituais, vinculados ao processo de cuidar, em disciplinas relacionadas ao cuidado no microcurricular, para verificar seu nível de inserção no planejamento curricular e transferência de conhecimento. para o aluno. Método: foi classificado como descritivo, de campo, transversal. A amostra foi composta por 29 docentes da área profissional. Foram elaborados dois instrumentos de coleta de dados: ficha de registro de 12 sílabas para a análise dos conteúdos do cuidado com vinculação dos teóricos e provas aos professores para confrontar sua utilidade nas aulas. Resultados: A revisão das sílabas mostrou que $21,43 \%$ incluem teorizadores e $78,57 \%$ não envolvem organização baseada em patologias clínicas e não em diagnósticos de enfermagem no planejamento das disciplinas. A aplicação do teste mostra um domínio médio de $63,06 \%$ no conhecimento dos modelos conceituais, um domínio médio de $46,55 \%$ no conhecimento e uso de teorizadores no processo de cuidar, $39,65 \%$ corresponde ao envolvimento dos teorizar nos conteúdos das disciplinas seguido de $31,03 \%$ com baixo uso. Conclusões: Fica evidente a pouca inserção de teóricos de enfermagem, no Processo de Cuidado de Enfermagem na maioria das disciplinas da unidade profissional e nos guias práticos, o que dificulta a relação teoria-prática para se tornar enfermeiro.

Palavras-chave: Educação em enfermagem; professores de enfermagem; processo de enfermagem; modelos de enfermagem

\section{INTRODUCCIÓN}

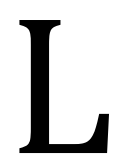
os programas o diseños curriculares, son estructuras académicas que orientan la gestión de formación profesional del estudiante, en la carrera de enfermería, el objeto de estudio es el cuidado siendo este el eje principal para el profesional que dirige sus esfuerzos a promover la calidad de vida de las personas que tiene a su cuidado, así la formación académica de enfermería a nivel de universidad tiene su vinculación en la década de los treinta del siglo XX en América Latina, con procesos de formación muy heterogéneos, es decir con diferentes programas y años de estudios, siendo esto un limitante en el reconocimiento social de la profesión.

Posner (1), plantea que el currículo responde a problemas previamente identificados, por lo tanto es el mediador entre la teoría y la realidad de la enseñanza, se establecen criterios que son organizados en el programa de asignatura y según

el Reglamento para la elaboración, implementación, seguimiento y evaluación al sílabo en su art. 2, define al programa de asignatura como "instrumento operativo de desarrollo para el logro de objetivos y operatividades en el microcurrículo, como acciones que orientan el aprendizaje en los estudiantes" (2).

Así, la planificación micro curricular es la unidad de aprendizaje más pequeña, abarca un tema completo; tiene unidad y coherencia en sí misma. El Reglamento para la elaboración, implementación, seguimiento y evaluación al sílabo, en su art. 3 define al silabo como "instrumento de planificación microcurricular que cumple la función de guía y orientación de los aspectos principales de desarrollo de la asignatura, además de ser un instrumento de transparencia susceptible de análisis, revisión, critica y mejoramiento" $(2,3)$.

Por su parte el Modelo Genérico de Evaluación del CEAACES, define al sílabo como" un instrumento de planificación de la 
enseñanza universitaria, que cumple la función de guía y orientación de los principales aspectos del desarrollo de una asignatura" (3), el mismo que debe guardar conexión lógica y funcional en la organización de los contenidos y acciones previstas en la enseñanza, es decir, constituye la acción pedagógica en las orientaciones del currículo.

Conceptualizado a enfermería como carrera de formación se la considera como ciencia al basarse en el método científico a través del proceso de atención de enfermería y como arte en el manejo de la creatividad en el cuidado, ya que engloba una serie de modelos conceptuales con visiones filosóficas denominadas modelos y teorías de enfermería, los mismos que están cada vez "más presentes en los programas formativos de enfermería, sobre todo a nivel de licenciatura" (4). Figueredo et al (5) expresa que estos modelos tienen un cuerpo de conocimientos que mejoran la práctica del personal de enfermería lo que permiten el desarrollo de la profesión.

Pero, a pesar de los artículos de investigaciones escritas alrededor de las teorías de Enfermería, aún persiste el divorcio entre los conceptos expresados por las teorizantes y la práctica del cuidado, según Rodríguez "no es nada saludable para el quehacer de la profesión" (6). Los fundamentos filosóficos, los modelos conceptuales y las teorías de enfermería son una necesidad no solo de formación educativa, sino que deben incorporarse a la práctica asistencial diaria del profesional, y así realice sus funciones sobre una base científica encaminado al objeto de la enfermería como es el cuidado del ser humano en todas sus dimensiones y ciclo de vida.

Uno de los problemas que enfrenta la profesión es la incongruencia relacionados con la teoría y la práctica que deben ser reflexionados, como, por ejemplo, la separación crónica entre estos dos aspectos (algunos autores (7-9), hablan de divorcio entre conocimiento y práctica), y la realidad frente al ideal de la Enfermería. En este sentido resulta indispensable consolidar la teoría y práctica para poder fundamentarse en un escenario objetiva y en las evidencias científicas. Se puede "apreciar la falta de concreción epistemológica en la Enfermería que da cabida a una ubicación errónea de la disciplina en la taxonomía científica" (9).

En consecuencia, el estudio tiene como objetivo determinar desde la academia la inclusión y utilización tanto en el aula como en las prácticas los diferentes modelos y teorías de enfermería, ligadas al proceso de atención de enfermería, a través de los contenidos de las asignaturas profesionalizantes (orientadas al conocimiento del campo de estudio y las áreas de actuación de la carrera), para verificar su nivel de inserción en la planificación y transferencia de conocimientos al estudiante.

Este estudio es importante para dar a conocer la aplicabilidad de los modelos conceptuales dentro del currículo y su utilización en el hacer enfermero, no deben de tener un impacto negativo; por lo que es necesario la búsqueda de estrategias que optimicen su utilidad para el mejoramiento de la profesión.

\section{MATERIALES Y MÉTODOS}

$\mathrm{L}$ a investigación se realizó en la Facultad de Ciencias de la Salud, carrera de Enfermería, se aplicó un estudio de tipo descriptivo, de campo y de corte trasversal, la recolección de la información se realizó mediante la revisión y análisis de los contenidos de las asignaturas relacionadas al cuidado como criterio de 
inclusión que aplican modelos conceptuales dentro del microcurrículo de la carrera de enfermería revisándose 12 sílabos: de las siguientes disciplinas enfermería básica, enfermería salud pública, enfermería clínico quirúrgica, enfermería gineco obstétrica, enfermería pediátrica, enfermería en salud mental, cuidados intensivos 1 y 2, y las asignaturas de los 4 ciclos de internado, para ello se aplicó el instrumento denominado FICHA DE REGISTRO DE SILABOS, la cual se apoya en el programa de asignaturas y en el análisis del seguimiento al silabo que aplica la carrera de enfermería, donde se pretendió verificar si los contenidos están sincronizados a la luz de las tendencias de la profesión, es decir si incorporan las teorizantes en la teoría y en la práctica dentro del microcurrículo de las asignaturas de la unidad profesional.

Asimismo, se utilizó un CUESTIONARIO A LOS DOCENTES estructurado por 10 ítems, con escala de 4 considerada la máxima puntuación, es decir alto, seguida de la escala 3 medio, escala 2 bajo y escala 1 muy bajo. El cual se aplicó a los 29 docentes de las asignaturas del área profesionalizantes, donde se buscó conocer el dominio de los modelos conceptuales en enfermería, del Proceso de Atención de enfermería, el involucramiento de las teorizantes en los contenidos de las asignaturas profesionalizantes, con un alfa de Cronbach igual a $0,984(98,4 \%)$, el instrumento tiene alta confiabilidad aceptable, por lo tanto, se puede aplicar.
En relación a las consideraciones éticas, el estudio fue totalmente voluntario, previa firma de consentimiento informado, se consideraron los principios de privacidad, honestidad, anonimato, confidencialidad a los encuestados, ambos instrumentos fueron validado por juicio de experto.

Para el análisis de los datos se procesaron a través del programa Excel (ficha de Sílabos) y el programa Spss versión 23 (cuestionario docente).

\section{RESULTADOS}

\section{Resultados de la ficha de registro del sílabo}

A continuación se describe el informe del análisis de la revisión de los 12 sílabos de las asignaturas que corresponde a la unidad profesional, de las cuales solo 3 entre ellas enfermería básica, enfermería pediátrica y ciclo clínico de internado rotativo que corresponde al 21,43\%, incluyen teorizantes, pero solamente en el aspecto teórico (en el aula de clase), más no en las prácticas clínicas, siendo el modelo más utilizado la valoración de Virginia Henderson con sus 14 necesidades y el modelo metodológico de Margaret Gordon, el resto de las 11 asignaturas, es decir el 78,57 \%, no utilizan las teorizantes de Enfermería ni en las clases teóricas, ni en las prácticas clínica. 


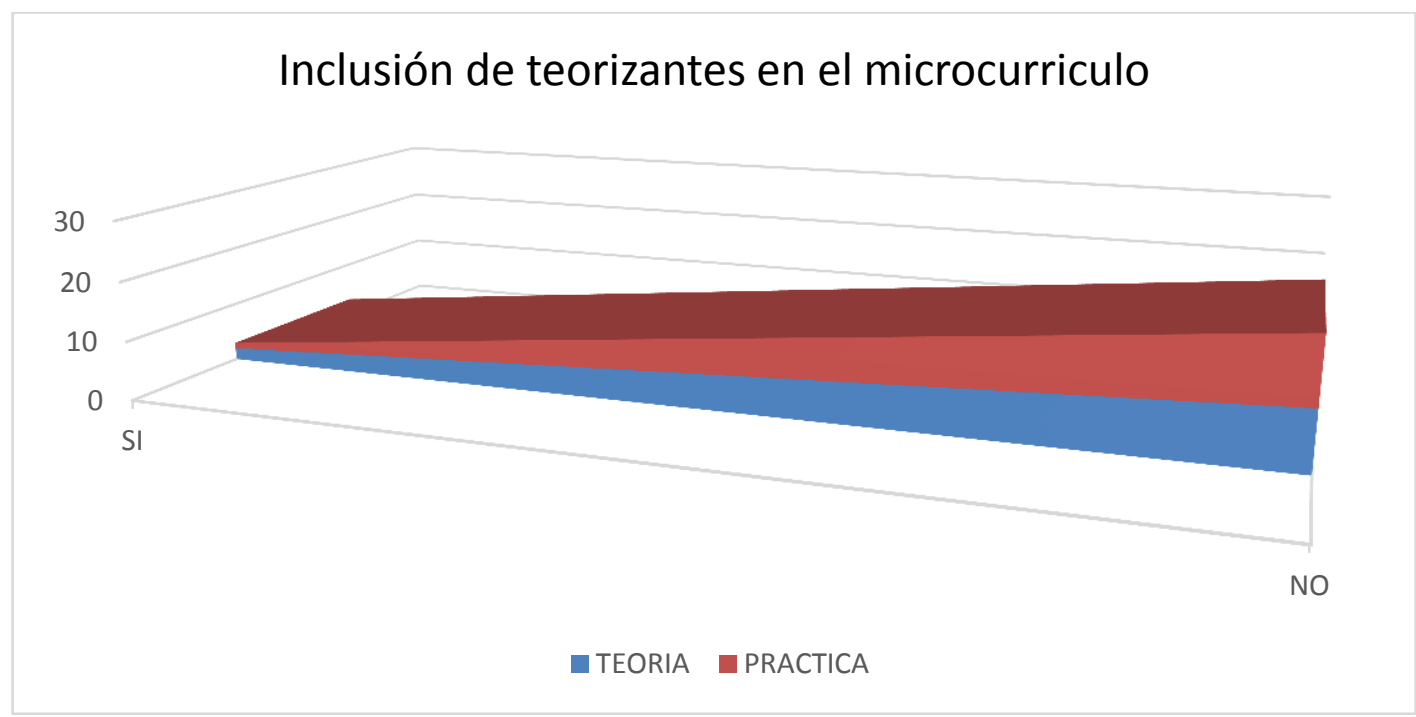

Figura 1. Nivel de inclusión de teorías de enfermería en el microcurrículo de las asignaturas del área profesional de la carrera de enfermería

La ficha de análisis del silabo que fue aplicado por la carrera de enfermería considera aceptable la relación de la naturaleza de los contenidos, que están programados y se sustentan en los conocimientos básicos sobre los que se fundamenta la carrera, los cuales se evidencian en los logros de aprendizaje, aunque se puede apreciar el mantenimiento del Modelo tradicional (enfoque biomédico), ya que los temas responden a las patologías clínicas y no a diagnósticos enfermeros.

\section{Resultados en relación al test docente}

Dominio y utilidad de modelos conceptuales en asignaturas de la unidad profesional

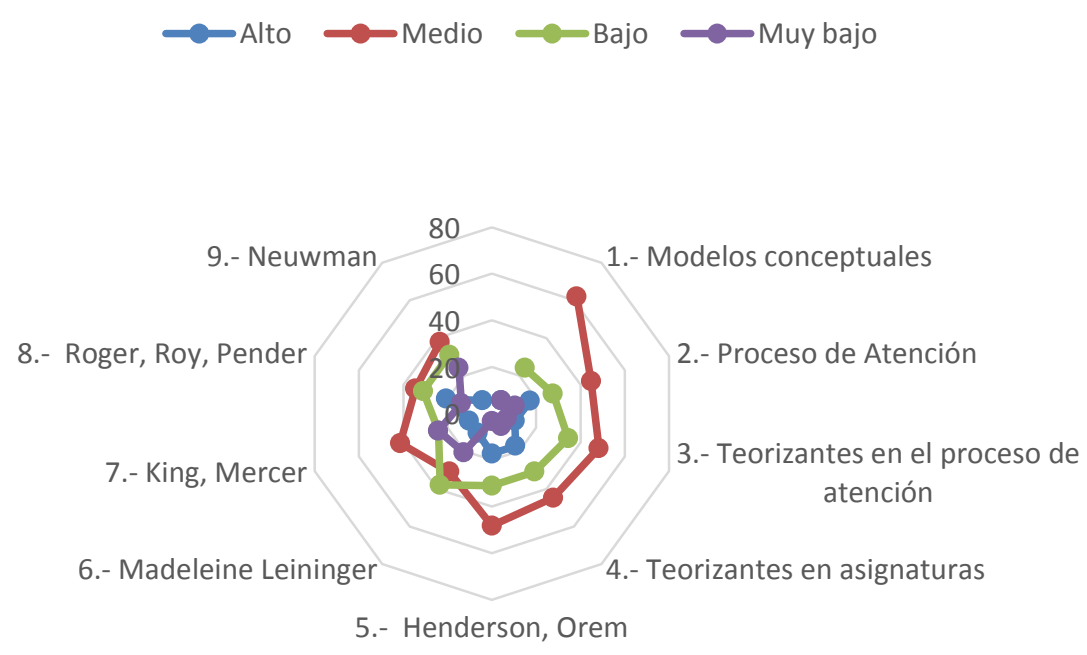

Figura 2. Dominio y utilidad de modelos conceptuales dentro del microcurrículo de la Carrera de Enfermería. 
En relación al dominio de los modelos conceptuales de enfermería los encuestados se ubicaron con el $62,07 \%$ lo que expresa un dominio medio con un nivel medianamente satisfactorio. En cuanto al aspecto dominio del proceso de atención de enfermería los datos evidenciaron el 44,83\% dominio medio por debajo del nivel de satisfacción.

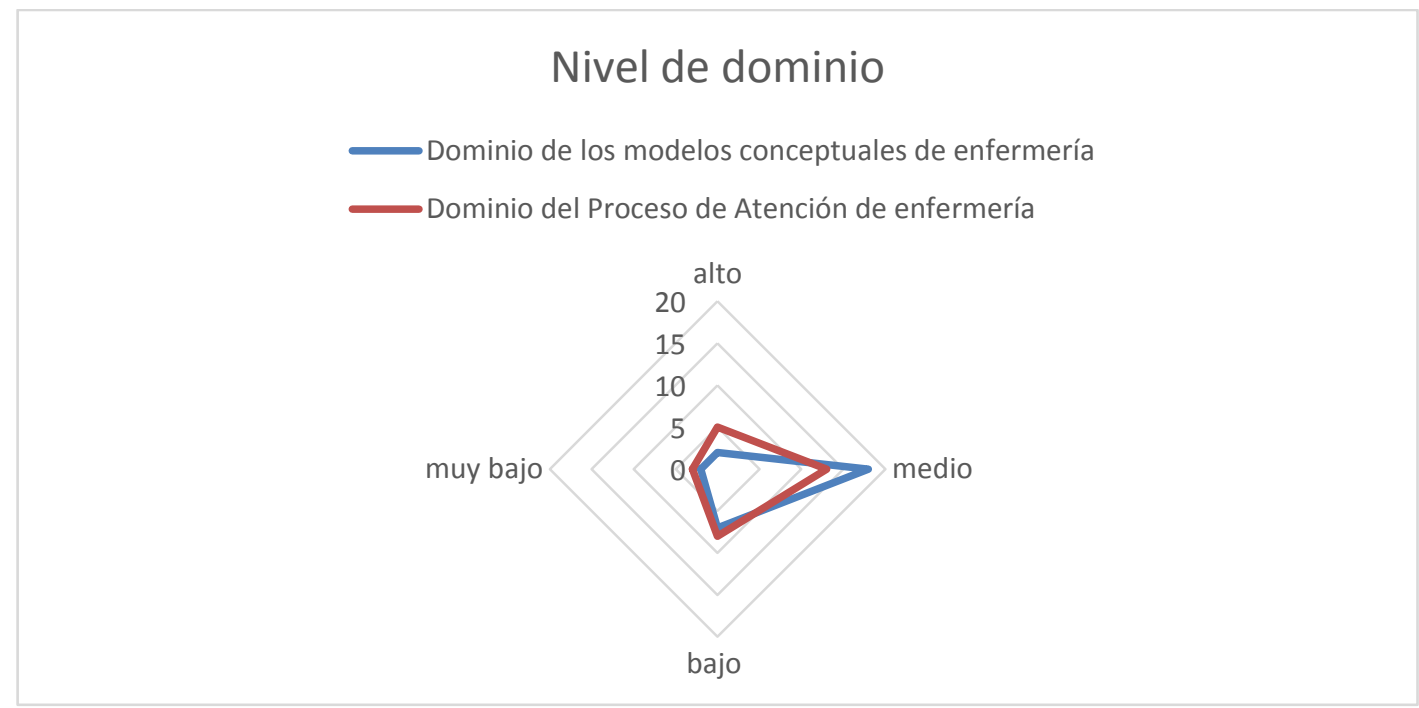

Figura 3. Nivel de dominio de modelos conceptuales y proceso de atención de enfermería

La utilización de las teorizantes en el proceso de atención de enfermería el 48,28\% lo emplea medianamente por debajo del nivel de satisfacción. El involucramiento de las teorizantes en los contenidos de las asignaturas profesionalizantes el 44,83\% es medio, por debajo del nivel de satisfacción.

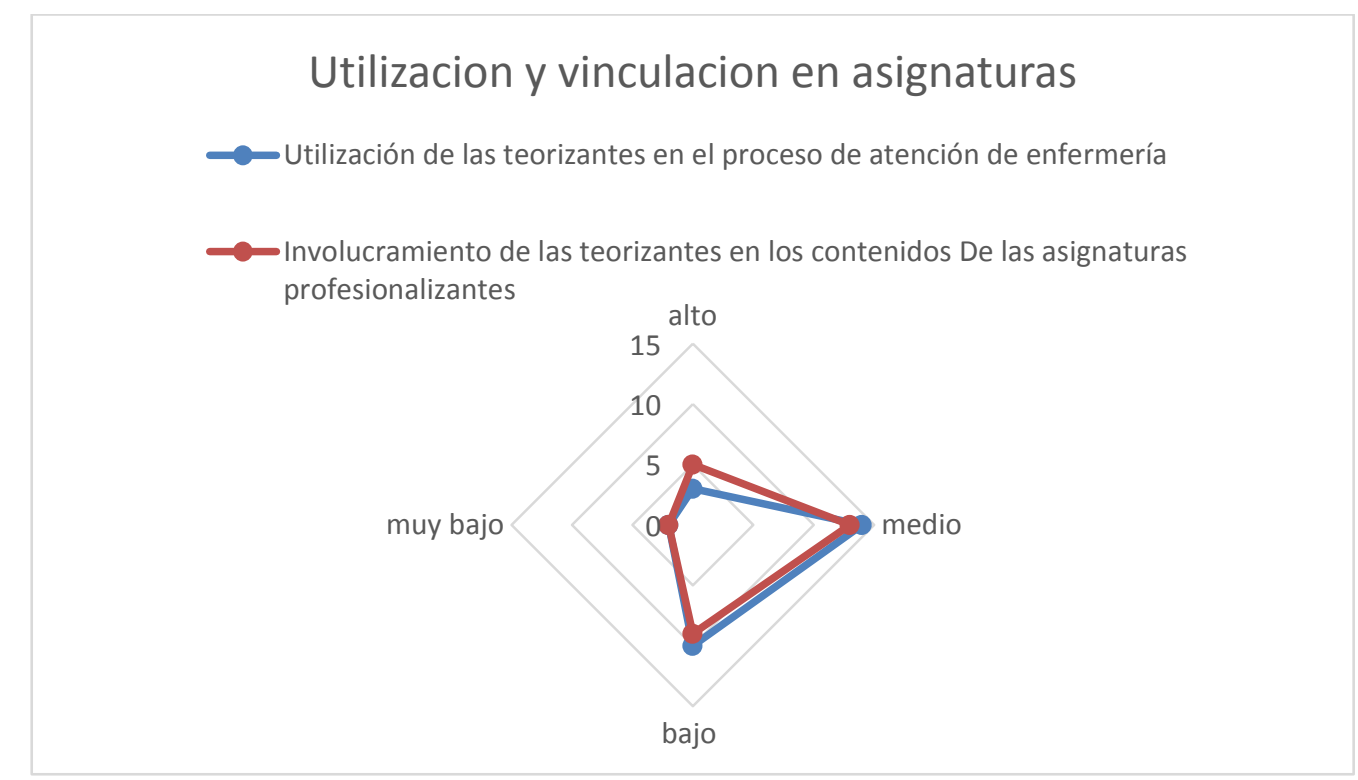

Figura 4. Utilización de teorizante y vinculación con los contenidos de las asignaturas del área profesional de la carrera de enfermería 
La utilización de estudios de casos con la aplicación de teorías de enfermería centrada en la resolución de las necesidades básicas (Henderson, Orem), se evidencio, el 48,28\% tienen un dominio medio por debajo del nivel de satisfacción. La aplicación de teorías de enfermería centradas en el cuidado cultural (Madeleine Leininger), los resultados expresan el $37,93 \%$ lo que evidencia una utilidad baja.

Cabe destacar que en la utilización de estudios de casos con la aplicación de teorías de enfermería centradas en la interacción
(King, Mercer), el 41,38 \% es medio por debajo del nivel de satisfacción. En la utilización de estudios de casos con la aplicación de teorías de enfermería centradas en los resultados (Roger, Roy, Pender), se mostró, el 34,48\% por debajo del nivel de satisfacción.

En tanto que en la utilización de estudios de casos con la aplicación de teorías de enfermería comunitaria (Neuwman), se evidenció que $37,93 \%$ por debajo del nivel de satisfacción.

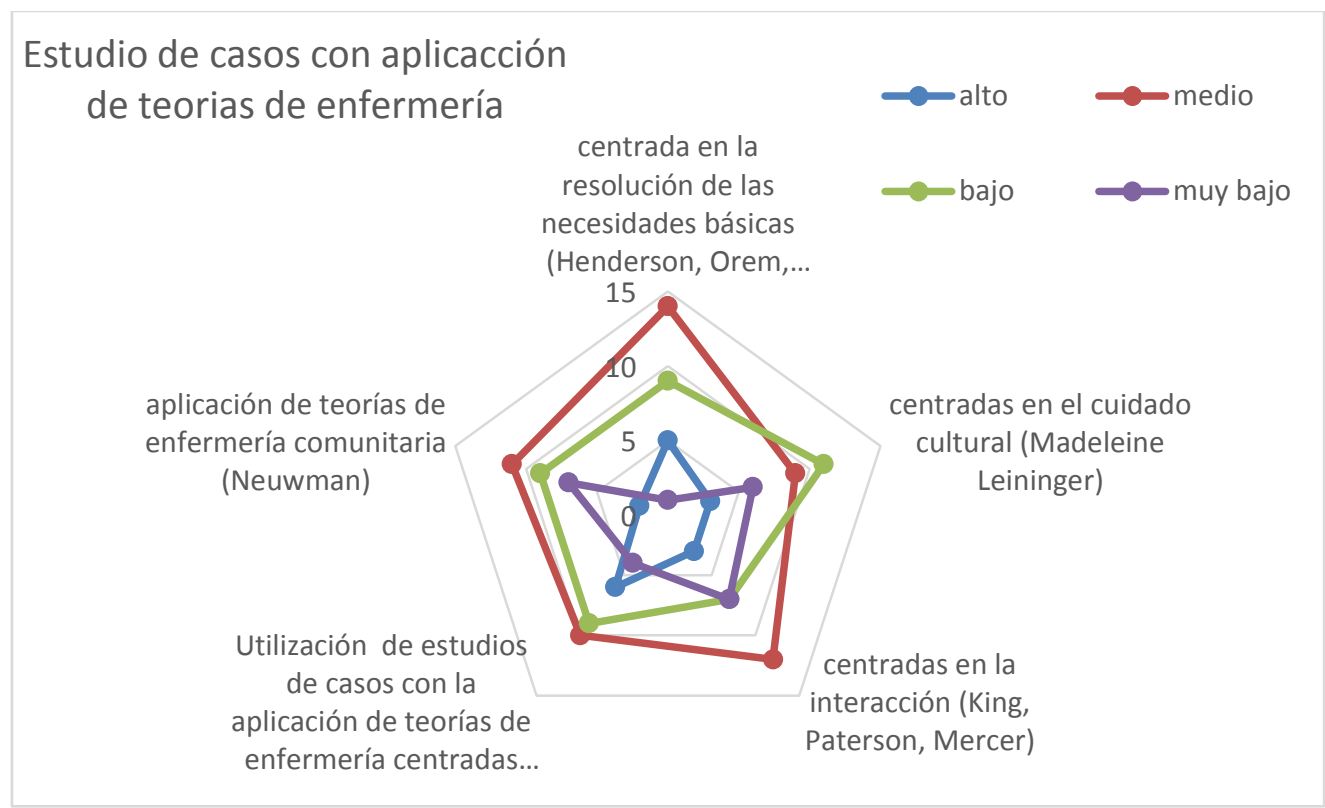

Figura 5. Utilización de estudios de casos con la aplicación de teorías de enfermería

\section{DISCUSIÓN}

$\mathrm{E}$ $n$ las asignaturas del primer semestre los estudiantes son orientados en el conocimiento general de las diversas teorías, generalidades que se pierden en el transcurso de formación, al no evidenciarse dentro del microcurrículo la inserción de las diferentes teorías que son las que direccionan la práctica en enfermería.
En las asignaturas enfermería pediátrica que se dicta en el quinto semestre se justifica la disciplina desde la teoría de Virginia Henderson con sus 14 necesidades, y como modelo metodológico el de Margaret Gordon, pero su utilidad queda solamente plasmada en el documento escrito, ya que no se implementa en la práctica.

En el ciclo clínico del programa de internado rotativo de la carrera, se aborda varias teorías que tampoco son vinculadas 
a la práctica, donde paradójicamente estas asignaturas se desarrollan en un $90 \%$ en el área hospitalaria.

En varias asignaturas dentro del silabo está considerado el proceso de atención de enfermería en algunos contenidos de las patologías cínicas, quirúrgicas y la presentación de estudios de casos bajo la valoración de patrones funcionales de Margaret Gordon, pero no se inserta las teorías y modelos conceptuales que direccionan la epistemología de la enfermería y en las prácticas clínicas solo se enfocan al cuidado en la satisfacción del componente biológico.

En las guías prácticas revisadas se encuentran estudios de casos clínicos con proceso de atención de enfermería bajo la valoración y cuidado basado en el modelo teórico de Margert Gordon, y muy pocos con el modelo conceptual de Virginia Henderson, es decir que en el área de investigación tampoco son utilizados las diferentes teorías o marcos conceptuales de la profesión.

El resultado se relaciona con la investigación realizada por Santos Ruiz, et al (10), en su artículo "Modelos teóricos y estudiantes de enfermería: aspectos motivacionales para su elección durante las prácticas clínicas" (9), al haber coincidencia en el modelo más utilizado siendo este el de Virginia Henderson, al tener mayores publicaciones relacionadas con planes de cuidado sobre todo asociada a taxonomía NANDA, NIC y NOC, lo que garantiza su utilidad práctica.

Mientras que en el TEST dirigido a los docentes se evidencia dominio medio del proceso de atención de enfermería y la utilización de las diversos modelos y teorías, lo que contradice lo verificado en los sílabos y en las guías prácticas, que no están insertados en el proceso formativo de la carrera de enfermería, lo que ha dificultado el aprendizaje de estos contenidos en los estudiantes, donde solo el 44,44\%, de las asignaturas profesionalizantes incluyen los modelos y teorías de enfermería en la parte teórica y práctica, con inclinación a patrones funciones y muy poco a 14 necesidades, mientras que $35,71 \%$, no utilizan las teorizantes de Enfermería ni en las clases teóricas, ni en las prácticas clínicas.

Estos resultados se justifican con lo expuesto por López, et al (11), al ser los docentes lo que mantiene la brecha entre teoría y práctica; y el estudiante al ir a la práctica clínica se encuentra que el personal de enfermeras/os asistenciales no ejecutan la aplicabilidad de los modelos teóricos en el trabajo diario, quizás debido a que los modelos conceptuales no se incluyen en el currículo de pregrado lo que ha provocado una generación de enfermeras/o en activo que no está adecuadamente formada en la vinculación de teorizantes dentro del proceso atención de enfermería.

Se relaciona además con la investigación de revisión integrativa de publicaciones de Figueredo et al (5) al concluir la existencia disociativa entre la teoría y la práctica, algunas enfermeras $\mathrm{y}$ enfermeros no reconocen su aplicabilidad al considerarlos cuerpos teóricos presentes dentro de la formación, pero ausente en la parte clínica.

\section{CONCLUSIONES}

nivel curricular es notorio la
falencia de la inclusión de los
modelos conceptuales vinculados en el proceso de atención de enfermería y del dominio de los docentes en su articulación, comprobado a través de los procesos metodológicos el bajo nivel de inserción en la planificación microcurricular y la poca transferencia de conocimientos en el estudiante.

Por lo que se determina la poca inclusión de las teorizantes de enfermería, 
en el Proceso de Atención de Enfermería en la mayoría de las asignaturas de la unidad profesional y en las guías prácticas, lo que dificulta la relación teoría - practica del hacer enfermero. Los resultados ponen en evidencia la escasa adquisición de los conocimientos científicos propios de enfermería, con la consecuencia de fallas en la aplicación de estos conocimientos científicos durante su desempeño profesional lo cual estaría incidiendo de forma negativa en el cuidado que se brinda al paciente; un cuidado empírico, rutinario e impersonal.

- Declaración conflicto de intereses

- Las autoras manifiestan no presentar conflicto de intereses.

\section{REFERENCIAS BIBLIOGRÁFICAS}

1. Posner, George. Análisis del currículo. México: McGraw Hill. 2005

2. Facultad de Ciencias de la Salud. Reglamento para la elaboración, implementación, seguimiento $y$ evaluación al sílabo de. Babahoyo: Universidad Técnica de Babahoyo. 2015

3. CEAACES. Modelo Genérico de Evaluación. Quito: Consejo de Educación Superior. 2015

4. León Román CA. Nuevas teorías en enfermería y las precedentes de importancia histórica, Rev Cubana Enfermer [Revista en Internet], 2017, (citado 6 ener 2020); 33(4). Disponible en: https://cutt.ly/tlra55U

5. Figueredo Borda Natalia, Ramirez Pereira Mirliana, Nurczyk Selene, Diaz Videla Valeria. Modelos y teorías de Enfermería: sustento para los cuidados paliativos, Enfermer: Cuidados humanizados, [Revista en Internet], 2019, (citado 4 feb 2020);
8(2). Disponible en: https://cutt.ly/Hlrsb5Q

6. Rodríguez Martínez R. Modelos de enfermería integrados al cuidado del paciente nefrológico en el contexto cubano. Rev Cubana Enfermer [Revista en Internet], 2012, (citado 20 feb 2020); 28(№ 4). Disponible en: http://scielo.sld.cu/scielo.php?script= sci_arttext\&pid=S0864-

03192012000400004

7. Mejía Lopera María Eugenia. Reflexiones sobre la relación interpersonal enfermera-paciente en el ámbito del cuidado clínico. Index Enferm [revista en la Internet]. 2006 [citado 12 Mar 2020]; 15(54): 48-52. Disponible en:

http://scielo.isciii.es/scielo.php?script $=$ sci_arttext $\&$ pid $=S 113212962006000$ 200010\&lng=es

8. Silva de Andrade L, Viana L. Reflexiones sobre el paradigma de la complejidad y el caos y sus nexos con la Enfermería del tercer milenio. Enferm Global [revista en la Internet]. 2008 [citado 21 Mar 2020];7(3)1-5. Disponible en:

https://revistas.um.es/eglobal/article /view/36261/34751

9. Sancho Cantus, D., Prieto Contreras. Teorías y modelos en la práctica enfermera. ¿Un binomio imposible? Enferm Global [revista en la Internet]. 2012 [citado 25 Mar 2020];11(27): 292-298. Disponible en: http://scielo.isciii.es/scielo.php?script =sci_arttext\&pid=S169561412012000300015

10. Santos Ruiz S, López Parra M, Sánchez Rueda G, Bardallo Porras L, Fernández Donaire L, Zabalegui Yárnoz A, Tomás Sábado. Modelos teóricos y estudiantes de enfermería: aspectos motivacionales para su elección 
durante las prácticas clínicas. Educación Médica, [revista en la Internet]. 2011 [citado 28 Mar 2020]; 14(4). Dispoible en:

http://scielo.isciii.es/scielo.php?script =sci_arttext\&pid=S1575-

18132011000200008

11. López Parra M, Santos Ruiz S, Varez Peláez S, Abril Sabater D. Reflexiones acerca del uso y utilidad de los modelos y teorías de enfermería en la práctica asistencial. Enfermer Clínic, revista en la Internet]. 2006 [citado 3 abr 2020];16(4):218 - 221. Disponible en: https://www.elsevier.es/esrevista-enfermeria-clinica-35articulo-reflexiones-acerca-del-usoutilidad-13091857 